\title{
Characterization of joint roughness using spectral frequencies and photogrammetric techniques
}

\author{
Ramiro García-Luna, Salvador Senent and Rafael Jimenez \\ Universidad Politécnica de Madrid, Madrid, Spain \\ ETSI Caminos, C. y P.; C/ Profesor Aranguren s/n; 28040 Madrid; Spain. \\ r.gluna@upm.es
}

\begin{abstract}
In this study we propose a novel methodology to characterize the roughness of rock joints using photogrammetry techniques and frequency analyses. The process starts with the generation of a 3D model (a dense point cloud) using the Structure from Motion (SfM) technology. Joint profiles are then extracted from this model and their frequency spectra are obtained using the Fast Fourier Transform. Finally, several approaches to parametrize the amplitude-frequency relationships are proposed, so that the roughness of the joint profiles are characterized with the fitting parameters. The methodology can differentiate between waviness and roughness, so that it could be used in future analysis of the shear behaviour of joints affected by this distinction. The proposed methodology is applied to two samples of granite representative of an unweathered fresh-cut surface with natural roughness. The results from the parametrization of the frequency spectra are used to carry out a classification analysis to study if their best-fitting parameters can estimate the Joint Roughness Coefficient (JRC) adequately. Results show that JRC values estimated with the spectral information tend to be quite similar (with errors less or equal to \pm 2 in about $80 \%$ of cases) to those estimated using the $\mathrm{Z}_{2}$ statistical parameter, therefore validating the use of frequency spectra to characterize the roughness of rock joints.
\end{abstract}

Keywords: Joint Roughness Coefficient (JRC), Frequency spectra, Structure from Motion, Rock joints roughness, Fast Fourier Transform.

\section{Caracterización de la rugosidad en juntas mediante análisis frecuencial y técnicas fotogramétricas}

\begin{abstract}
RESUMEN
En este trabajo se propone una metodología novedosa para caracterizar la rugosidad de las juntas de roca mediante técnicas fotogramétricas y análisis frecuencial. El proceso comienza con la generación de un modelo $3 D$ (una nube de puntos densa) utilizando la técnica Structure from Motion (SfM). A continuación, se extraen del modelo los perfiles de rugosidad y se obtienen sus espectros de frecuencia mediante la Transformada Rápida de Fourier. Finalmente, se proponen varias ecuaciones para parametrizar las relaciones amplitud-frecuencia, de manera que la rugosidad de los perfiles queda caracterizada con los parámetros de ajuste. La metodología permite diferenciar entre ondulación y rugosidad, de modo que puede emplearse en futuros análisis sobre la caracterización de la resistencia al corte de las juntas cuyo comportamiento se ve afectado por esta distinción. A partir de los resultados de la parametrización de los espectros de frecuencia, se lleva a cabo un análisis de clasificación para analizar si los parámetros de ajuste permiten estimar adecuadamente el coeficiente de rugosidad (JRC). Los resultados muestran que los valores del JRC obtenidos a partir del espectro de frecuencias son bastante similares (con errores menores o iguales a \pm 2 en aproximadamente el $80 \%$ de los casos) a los estimados usando el parámetro estadístico $\mathrm{Z}_{2^{\prime}}$ validando así el uso del análisis frecuencial para caracterizar la rugosidad de las juntas de las rocas.
\end{abstract}

Palabras clave: Coeficiente JRC, Espectro de frecuencias, Structure from Motion, Rugosidad de juntas en roca, Transformada Rápida de Fourier. 


\section{Introduction}

Joint roughness is a key parameter affecting joint strength (see e.g., Patton, 1966; Barton and Choubey, 1977). The Joint Roughness Coefficient (JRC), with values between 1 and 20, and used in the joint strength criterion of Barton and Choubey (1973) is probably the most common tool for assessing the influence of joint roughness in practice. However, its visual assessment is subjective, and several methods have been proposed to correlate JRC with other (objective) statistical parameters that characterize the joint profile (see e.g., Tse and Cruden, 1979; Yu and Vayssade, 1991), to characterize joint roughness with fractal approaches (see e.g., Ge et al., 2014; Li and Huang, 2015), or to characterize joint roughness using its spectral frequencies or a wavelet analysis (see e.g., Chae et al., 2011, Zou et al., 2015). One main advantage of these techniques is that they differentiate between waviness (at a large scale; also referred to as undulation) and smoothness (at a smaller scale; also referred to as unevenness or second-order roughness): since joint behavior is controlled by waviness or smoothness depending on the normal tension or the shear displacement (ISRM, 1978) and other approaches that characterize roughness using only one parameter are unable to account for it (Yang et al., 2001).

In this paper we propose a methodology to quantify joint roughness (at both scales) using the spectral frequencies of its profile. Although such a profile can theoretically be captured using any technique that provides continuous profiles of the joint (e.g., with 3D laser scanner (LiDAR), or even with contour gauges or profilometers), we focus on the remote 3D characterization techniques that measure joint roughness using digital photogrammetry and digital image processing (Unal and Unver, 2004), as shown by previous successful applications in the laboratory and the field (Wernecke and Marsch, 2015; Tatone and Grasselli, 2013).

In particular, we have used the Structure from Motion (SfM) technology, a technique that does not require special cameras, generating $3 \mathrm{D}$ point clouds using information from photographs and a devoted software, to construct 3D models of the joint surfaces, from which roughness profiles are extracted. The spectral frequencies of such profiles are then obtained and approximated using suitable mathematical (exponential or straight) functions that allow us to define the roughness profiles using only a few parameters. The methodology is finally demonstrated using two samples of granite.

\section{Generation of 3D models of rock joints using SfM}

To be able to locate points in the model, traditional photogrammetry (see e.g., Slama et al., 1980) requires precise information about (i) location and orientation of the camera; and (ii) the location in 3D of several control points that appear in the photographs. The a)

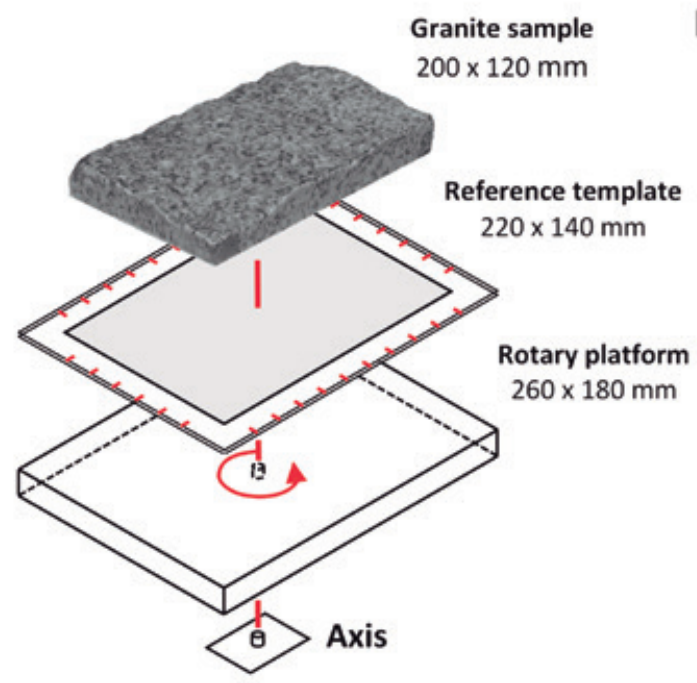

b)

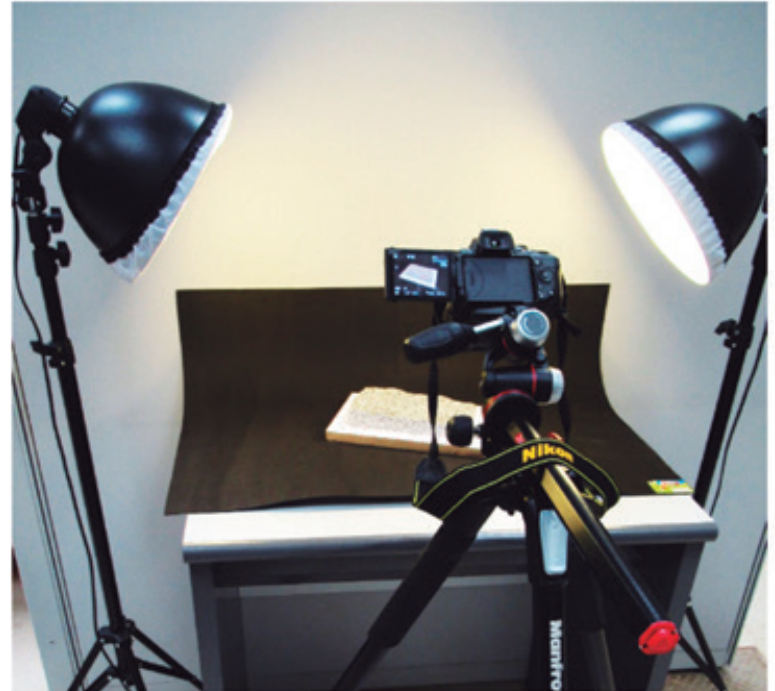

Figure 1. Equipment: (a) rotary platform with the reference template; (b) macro-photography set-up.

Figura 1. Equipamiento: (a) plataforma para la muestra, (b) set de fotografía macro. 
main advantage of the SfM technique is that it can use several overlapping photographs to generate $3 \mathrm{D}$ models of the scene without the need to know the 3D coordinates of the control points. Location and orientation of the camera are not required either, as this information is extracted from the analysis of available data.

\section{Equipment}

As indicated above, the traditional SfM technique uses several overlapping images, taken from different positions around the object of interest, to develop a 3D model ("a point cloud") of the object. In this study, we have changed this traditional set-up and used a rotary platform (of $260 \mathrm{~mm} \times 180 \mathrm{~mm}$ dimensions) on top of which the granite samples are laid. The camera, a Nikon NIKKOR AF-S DX 18-55 mm F 3.5-5.6G VR with $24 \mathrm{MP}$, is installed on a professional Manfrotto tripod (with high weight and adjustable height), and a remote-control cordless shooting device is used. Two lights illuminate the sample from opposite sides, and an opaque dark background (similar to a cyclorama) has been created using black paper. (See Fig. 1.)

This set-up allows us to take many photographs in a short time, without re-focusing the camera and without problems due to vibration of the camera or shadows on the surface. In particular, this set-up allows us to fully cover the sample surface with photographs that have a high degree of overlapping (approx. 95\%).

To provide a reference to scale the model, we have also used a template with a rectangular reference frame, with known dimensions of $220 \mathrm{~mm} \times 140 \mathrm{~mm}$ (see Figs. 1 and 2). This reference frame incorporates 38 ground control points or GCPs (one every $2 \mathrm{~cm}$ )

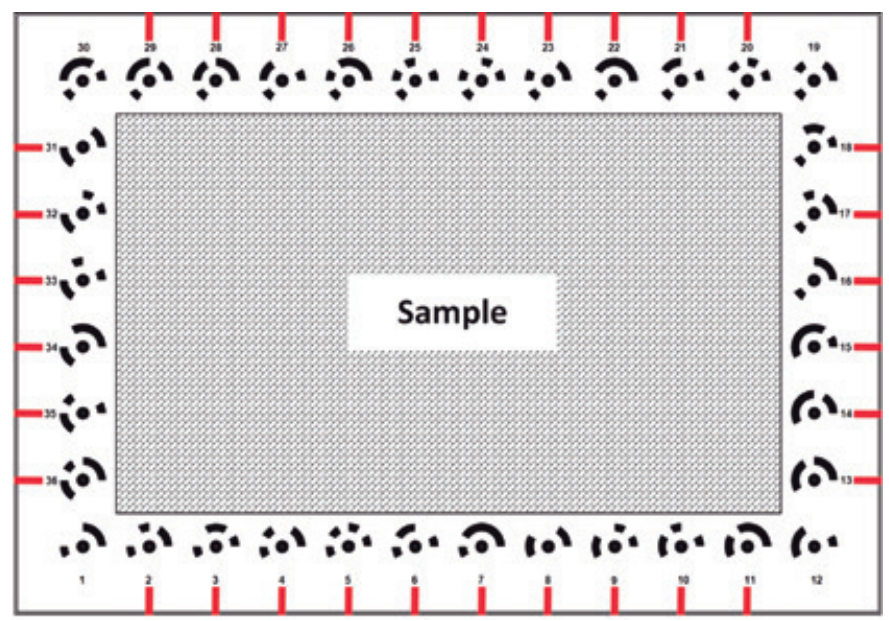

Figure 2. Illustration of the reference template.

Figura 2. Plantilla de referencia. along its boundaries. GCPs are points that can be recognized in the photographs and whose coordinates are known. They allow us to (i) correctly scale the model and (ii) to set-up a horizontal $(x-y)$ reference system that will be used in later analyses (it helps us to locate the position of the roughness profiles).

\section{Digital processing of photographs}

There are several types of software, e.g., PhotoModeler (Microsoft), ReMake (Autodesk), PhotoScan (Agisoft), Pix4D (Pix4D SA) which use the SfM technique to develop 3D models of objects. We have use the professional version of Agisoft PhotoScan (LLC, 2016) in this study. (Note that the standard version does not allow the use of GCPs and they are required to scale the generated 3D models.)

Agisoft PhotoScan uses the SfM technique by means of the following steps: (i) it identifies multiple key points in each photograph; (ii) it matches them (in overlapping images); (iii) it uses an iterative adjusting algorithm to estimate the camera parameters for each photograph, so that the 3D position of the key points are computed, leading to an initial disperse 3D point cloud; (iv) it constructs a high density 3D point cloud using multi-view stereo (or MVS) techniques; and, finally, (v) it scales and orientates the point cloud within a reference system using the ground control points. (For a better performance, it is recommended that GCPs are visible within at least three photographs; see LLC, 2016.)

The coordinates of the 3D points cloud generated with Agisoft PhotoScan are then transferred to MATLAB (The MathWorks, Inc., 2019), a mathematical system for data representation and analysis. As is explained below, MATLAB is used to plot and extract the roughness profiles from the 3D point cloud of the sample and for the posterior frequency analysis.

\section{Characterizing joint roughness using spectral analysis}

\section{Obtaining spectral frequencies from joint profiles}

If we consider the joint profile as a spatial signal, we can use signal processing techniques, such as spectral representation or filters. The frequency spectrum can then be obtained using the following formula (Prandoni and Vetterli, 2008):

$$
Z[k]=\sum_{n=0}^{N-1} Z[n] \cdot e^{j \frac{2 \pi}{N} n k} \text { with } k=0,1, \ldots, N-1
$$

where $z$ represents the amplitudes of the profile at the sampled points ( $N$ is the number of sampled 
points) and $Z$ represents the spectral amplitudes. Note also that $e^{j \frac{2 \pi}{N} n k}$ represent elements, $w_{n}^{(k)}$, that form an orthogonal basis in $\mathrm{C}^{\mathrm{N}}$, as:

$$
W_{n}^{(k)}=e^{j}{ }_{N}^{2 \pi} n k
$$

Figure 3(a) shows an example of a joint profile (obtained from the application example that will be described below and Figure 3(b) shows its corresponding frequency spectrum with low frequencies (on the left) representing the waviness and high frequencies (on the right) representing the smoothness. Note that only positive frequencies are considered, and that the joint, like any other continuous signal, must be sampled before the analysis, so that the maximum observed frequency will be one half of the sampling frequency (Prandoni and Vetterli, 2008).

\section{Parametrization of the frequency spectrum}

To parametrize the frequency spectra, we must seek the type of mathematical function that best represents the amplitude-frequency relationships obtained from

a)

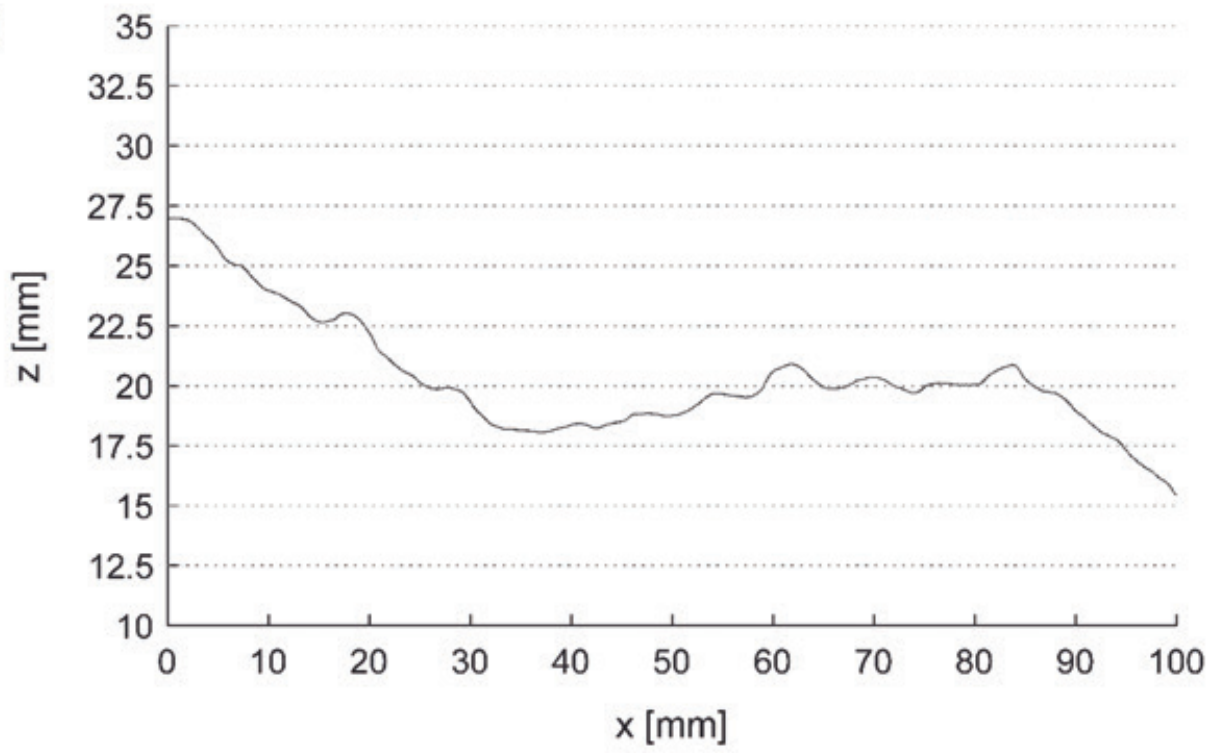

b)

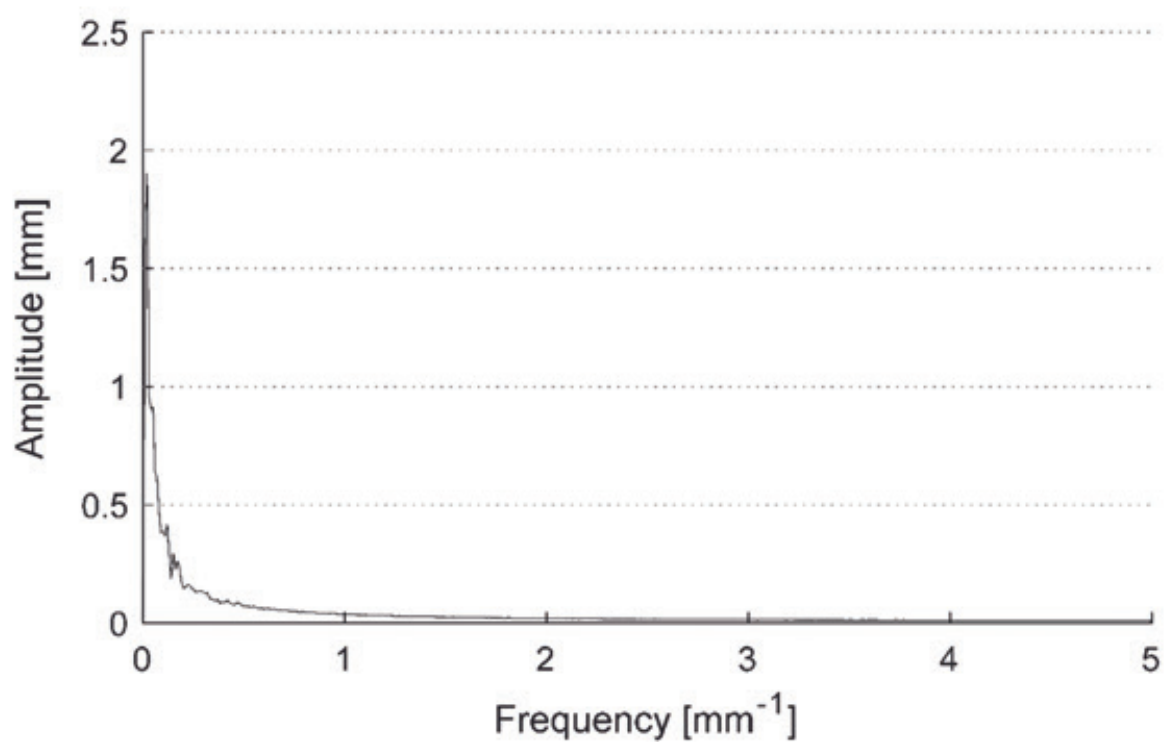

Figure 3. Example of (a) roughness profile, and of (b) its frequency spectrum.

Figura 3. Ejemplo de (a) perfil de rugosidad, y de (b) su espectro de frecuencias. 
the spectral analysis. To that end, and given its influence on the shear behaviour of the joints, it seems appropriate to establish a threshold value (i.e., a frequency) that separates the waviness and the smoothness, and that allows us to adjust one specific function to each part of the spectrum. Such functions can be independent (i.e., not having continuity at the threshold), or they can be constrained so that continuity at the threshold is fulfilled.

Although this threshold will depend on the problem being analyzed (hence requiring a more in-depth and case-by-case analysis), we can use the reference values proposed in the literature as guidance. For instance, Palmström (2001) proposes a threshold between waviness and smoothness of around $0.05-0.50 \mathrm{~m}$ for roughness profiles with lengths of up to $10 \mathrm{~m}$, or around $1 / 200-1 / 20$ of the profile length. For a particular profile, we have used a threshold defined as the profile length divided by 50 , and transformed it into a frequency using its inverse.

Based on this description, and considering that the best approach to fit the spectrum is not known in advance, we propose five different approaches to parametrize the amplitude-frequency relationships of the frequency spectra, as follows:

(1) Approach E: fitting with a unique negative exponential function $\left(Z=K \cdot e^{-\lambda f}\right)$ that covers the whole spectrum; where $f$ is the frequency and $Z$ is the spectral amplitude, and where $K$ and $\lambda$ are the fitting parameters.

(2) Approach EEi: fitting with two independent negative exponential functions, one for high frequencies $\left(Z=K_{h} \cdot e^{-\lambda_{h}}\right)$ and one for low frequencies $\left(Z=K_{l} \cdot e^{-\lambda_{l}}\right)^{f}$, where $\left(K_{p} \lambda_{l}\right)$ and $\left(K_{h^{\prime}} \lambda_{h}\right)$ are the fitting parameters for low and high frequencies respectively.

(3) Approach EEc: fitting with two negative exponential functions for high and low frequencies $\left(Z=K_{h} \cdot e-\right.$ $\lambda_{h} f$ and $\left.Z=K_{l} \cdot e^{-\lambda l^{f}}\right)$ that are constrained to be continuous at the threshold between the high and low frequencies.

(4) Approach ESi: fitting with a negative exponential function for low frequencies $\left(Z=K_{1} \cdot e^{-\lambda f_{1}}\right)$ and an independent straight line for high frequencies $\left(Z=p_{1}+p_{2} \cdot f\right)$, where $p_{1}$ and $p_{2}$ are the fitting parameters.

(5) Approach ESc: fitting with a negative exponential function for low frequencies $\left(Z=K_{1} \cdot e^{-\lambda_{f} f}\right)$ and a straight line for high frequencies $\left(Z=p_{1}+p_{2} \cdot f\right)$. Both functions are constrained to be continuous at the threshold between the high and low frequencies.

Approach $E$ is the simplest one because, although it uses a negative exponential function with only two fitting parameters that generally agree well with the shape of amplitude-frequency relationships, it does not differentiate between the low and high frequencies (i.e., between waviness and smoothness). Approaches EEi and EEc do differentiate between high and low frequencies: EEi requires four parameters to be fitted $\left(K_{p} \lambda_{p} K_{h}, \lambda_{h}\right)$, whereas in EEc one of the parameters is dependent on the others due to the imposed continuity condition, so that only three parameters need to be fitted. Approaches ESi and ESc are similar, with the only difference being that they simplify the fitting function for high frequencies, using a straight line instead of a negative exponential.

\section{Application example}

The proposed methodology has been used to characterize two samples of granite. Next, we present details about the samples used, as well as about the procedure for generating the 3D models with the SfM technique and characterizing their roughness profiles using their frequency spectra.

\section{Description of samples}

The two granite samples used come from the same granite block, with a fine to medium grain size (crystals of 1-5 mm). They represent an unweathered freshcut surface with natural roughness. To facilitate their manipulation in the laboratory, the samples were cut with a widia rock saw, forming small rectangular samples with approximated dimensions of $200 \mathrm{~mm}$ length x $120 \mathrm{~mm}$ width $\times 40 \mathrm{~mm}$ height. (See Fig. 4).

a)

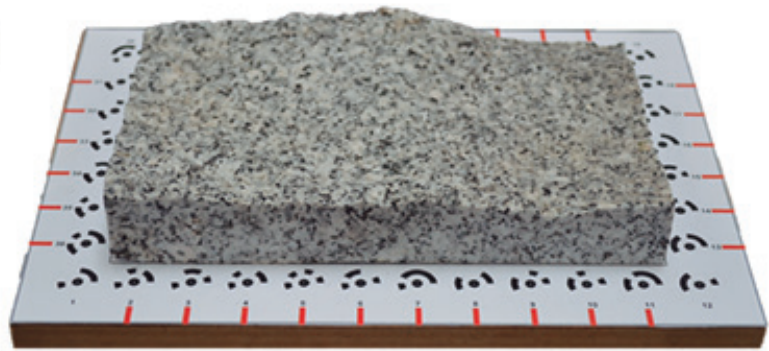

b)

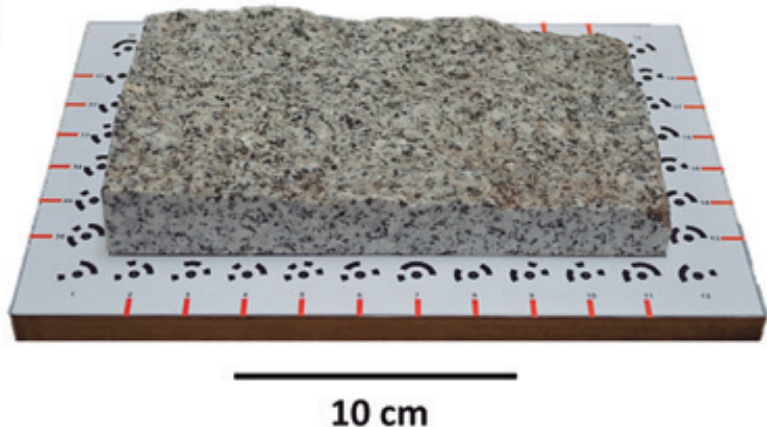

Figure 4. Granite samples employed in the analyses: (a) Sample 1; (b) Sample 2.

Figura 4. Muestras de granito empleadas en el análisis: (a) Muestra 1; (b) Muestra 2. 

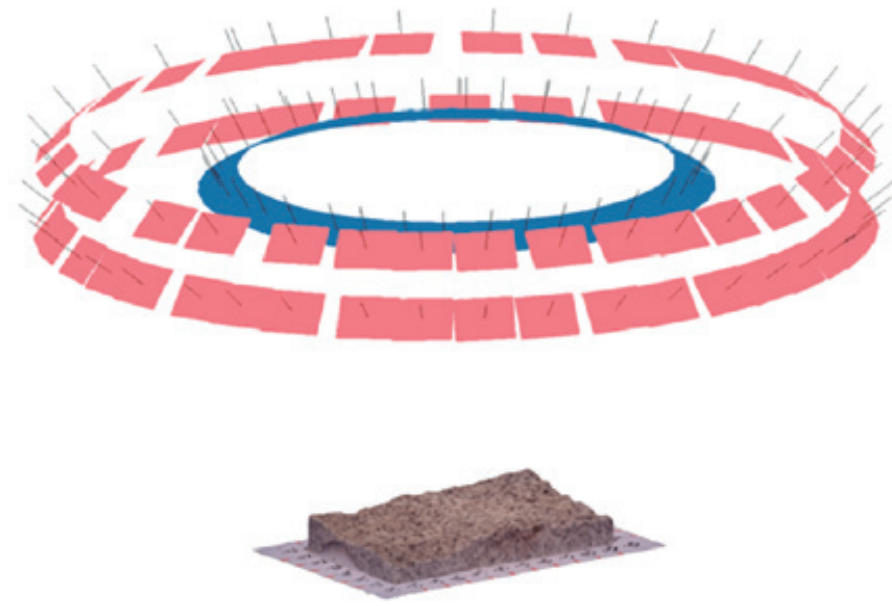

Figure 5. Camera positions for photographs used to construct the $\mathrm{SfM}$ model of Sample 1. (Different focal distances are indicated with different colours: pink for higher and blue for lower focal distances.)

Figura 5. Posiciones de la cámara en las fotografías empleadas para la generación del modelo SfM de la Muestra 1. (Las diferentes distancias focales se indican con diferentes colores: rosa para las distancias focales mayores y azul para las distancias focales menores).

\section{Point-cloud model}

We developed two photogrammetric models (Model 1 for the first sample and Model 2 for the second sample) using 110 photographs for each one. The process is described below.

\section{Data acquisition}

The photographs needed to construct the models were taken, with the aid of the rotary platform described above, from three different camera positions (i.e., with three different angles of the camera lens with respect to the sample). Three consecutive passes of $360^{\circ}$ were performed, taking photographs at intervals of approximately $10^{\circ}$. (As an example, Figure 5 shows camera positions during the photography of Sample 1.)
The photographs were taken using a fixed focal distance and fixed camera adjustments based on the available lighting conditions of the set. Specifically, all the photographs were taken with the maximum possible resolution (24 MP, 3:2; effective pixels $6000 \times 4000$; JPEG format) and with the following adjustments: manual mode, focal length $20-38 \mathrm{~mm}$, center-weighted average metering, MF, image stabilizer off, manual white balance, aperture F/25-32, exposure time $\leq 2$ " and ISO speed rating $100 / 21^{\circ}$.

Such adjustments were defined after some trials but before the actual photographs for each model were taken, and with the objective of providing an "optimum" configuration that homogenizes the focus and widens the depth of field, hence providing crisp images with great detail. In this way, we have minimized the operations needed between shootings, which are reduced to (i) rotating the sample (with the rotary platform) and (ii) shooting the camera (with the wireless shooting device). The use of the rotary platform provides significant time savings: the total time needed to prepare the camera and to take all photographs for each model was about 10 minutes, of which approximately $50 \%$ were used to adjust the camera parameters. Table 1 shows the focal distances and apertures used to take the photographs for each sample.

\section{Generation of the 3D model}

The first step of the process is to align and orientate the images and to generate an initial model: the disperse point cloud. However, this initial point cloud does not have the quality and precision required for the roughness analysis. Therefore, a second step is needed to generate a new point cloud with a much higher point density (similar to those provided by LiDAR devices) using MVS techniques. For both the disperse and high-density point clouds, Agisoft Pho-

\begin{tabular}{|c|c|c|c|c|c|}
\hline \multirow[b]{2}{*}{ Sample } & \multirow{2}{*}{$\begin{array}{l}\text { Average } \\
\text { distance to } \\
\text { the object }\end{array}$} & \multirow[b]{2}{*}{ Photos } & \multicolumn{3}{|l|}{ Camera settings } \\
\hline & & & $\begin{array}{l}\text { Focal length / } \\
\text { Equivalent in } 35 \mathrm{~mm} \text { format }\end{array}$ & Aperture & $\begin{array}{l}\text { Exposure } \\
\text { time }\end{array}$ \\
\hline \multirow{2}{*}{1} & \multirow{2}{*}{$39 \mathrm{~cm}$} & 36 & $20 \mathrm{~mm} / 30 \mathrm{~mm}$ & \multirow{2}{*}{ F 25} & \multirow{2}{*}{$1 / 1.3^{\prime \prime}$} \\
\hline & & 74 & $22 \mathrm{~mm} / 33 \mathrm{~mm}$ & & \\
\hline \multirow{2}{*}{2} & \multirow{2}{*}{$43.9 \mathrm{~cm}$} & 68 & $26 \mathrm{~mm} / 39 \mathrm{~mm}$ & F 29 & \multirow{2}{*}{$2^{\prime \prime}$} \\
\hline & & 42 & $38 \mathrm{~mm} / 57 \mathrm{~mm}$ & F 32 & \\
\hline
\end{tabular}

Table 1. Camera settings.

Tabla 1. Configuraciones de la cámara. 


\begin{tabular}{clclccc}
\hline \multirow{2}{*}{ Model } & \multicolumn{5}{l}{ Disperse point cloud } & \multicolumn{4}{l}{ High density point cloud } \\
\cline { 2 - 7 } & Accuracy & No of points & Accuracy & No of points & Ground resolution & Point density \\
\hline 1 & High & 6867 & High & 4279887 & $0.0742 \mathrm{~mm} / \mathrm{pix}$ & 45.4 points $/ \mathrm{mm}^{2}$ \\
2 & High & 6807 & Medium & 1547841 & $0.0597 \mathrm{~mm} / \mathrm{pix}$ & $17.5 \mathrm{points} / \mathrm{mm}^{2}$ \\
\hline
\end{tabular}

Table 2. Information about the point clouds used.

Tabla 2. Información sobre las nubes de puntos empleadas.

\begin{tabular}{cllll}
\hline \multirow{2}{*}{ Model } & \multicolumn{2}{l}{ Disperse point cloud } & \multicolumn{2}{l}{ High density point cloud } \\
\cline { 2 - 5 } & Accuracy & Time & Accuracy & Time \\
\hline 1 & High & $32 \mathrm{~min}$ & High & $1 \mathrm{~d} 12 \mathrm{~h} 40 \mathrm{~min}$ \\
2 & High & $42 \mathrm{~min}$ & Medium & $11 \mathrm{~h} 58 \mathrm{~min}$ \\
\hline
\end{tabular}

Table 3. Computation times to generate the photogrammetric 3D models in Agisoft PhotoScan.

Tabla 3. Tiempos de procesado para la generación de los modelos fotogramétricos 3D en Agisoft PhotoScan.

toScan allows the selection of different "levels of computation" that influence the precision and quality of the results (low, medium and high precision). Generating the high-density point cloud is the most computationally expensive step, taking about $95 \%$ of the processing time. For this reason, we worked with high-density point clouds generated with two levels: high for Model 1 and medium for Model 2 (see Table 2). This has allowed us to check whether the quality and precision of the results are reduced when a lower level model, with a reduced processing time $(<1 / 3)$, is used. Table 3 shows the computation times required to align and orientate the images and to generate the initial (disperse) point clouds, and to generate the final (high density) point cloud. (All computations were performed using a single core of an Intel Core i7-6700 computer [3.4GHz processor; $16 \mathrm{~GB}$ RAM] running the Windows operating system.)

Finally, the high-density point clouds generated for both models are scaled and orientated with Agisoft PhotoScan, with the aid of the relative coordinates of GCPs provided by the reference template. This scaling can also be verified, comparing the "real" distance between points in the template (measured with

\begin{tabular}{ccccc}
\hline Model & GCPs & $\begin{array}{c}\text { Xerror } \\
{[\mathrm{mm}]}\end{array}$ & $\begin{array}{c}\text { Yerror } \\
{[\mathrm{mm}]}\end{array}$ & $\begin{array}{c}\text { Z error } \\
{[\mathrm{mm}]}\end{array}$ \\
\hline 1 & \multirow{2}{*}{36} & 0.080 & 0.092 & 0.023 \\
2 & 0.062 & 0.064 & 0.057 \\
\hline
\end{tabular}

Table 4. Errors of ( $x, y$ and $z$ ) coordinates of the reference points of the template within the 3D model.

Tabla 4. Errores en las coordenadas $(x, y, z)$ de los puntos de referencia de la plantilla incluida en los modelos $3 D$. another measuring device, such as a precision rule) with the corresponding distances computed from the 3D model. The errors of the ( $x, y$, and $z)$ coordinates of reference points within the template (i.e., the differences between their real (known) coordinates and those provided by the 3D digital model) are listed in Table 4.

\section{Extraction of profiles}

The two 3D models generated with Agisoft PhotoScan (one for each sample) are then exported to MATLAB to (i) extract the roughness profiles and (ii) to conduct the frequency analysis. In particular, 58 profiles (37 transversal +21 longitudinal) of $10 \mathrm{~cm}$ length are extracted from the 3D model of each sample at distances of $0.5 \mathrm{~cm}$ between them. Out of these, 16 "validation profiles" (10 transversal + 6 longitudinal; at $2 \mathrm{~cm}$ separation between them) were selected to analyze the precision of the photogrammetric model and to analyze the goodness of the roughness characterization carried out with the remaining 42 profiles (referred to herein as "basic profiles").

\section{Analysis of the precision of the profiles}

To assess the precision of the photogrammetric models, we have used a "Barton-comb" (with needle separation of $1 \mathrm{~mm}$ ) to measure the roughness profiles of the samples at the locations of the validation profiles. As shown in Figure 6, where two of such profiles are compared, the results are practically identical.

We can use the average roughness $\left(R_{a}\right)$ of the difference profiles (calculated as the difference between the 
a)

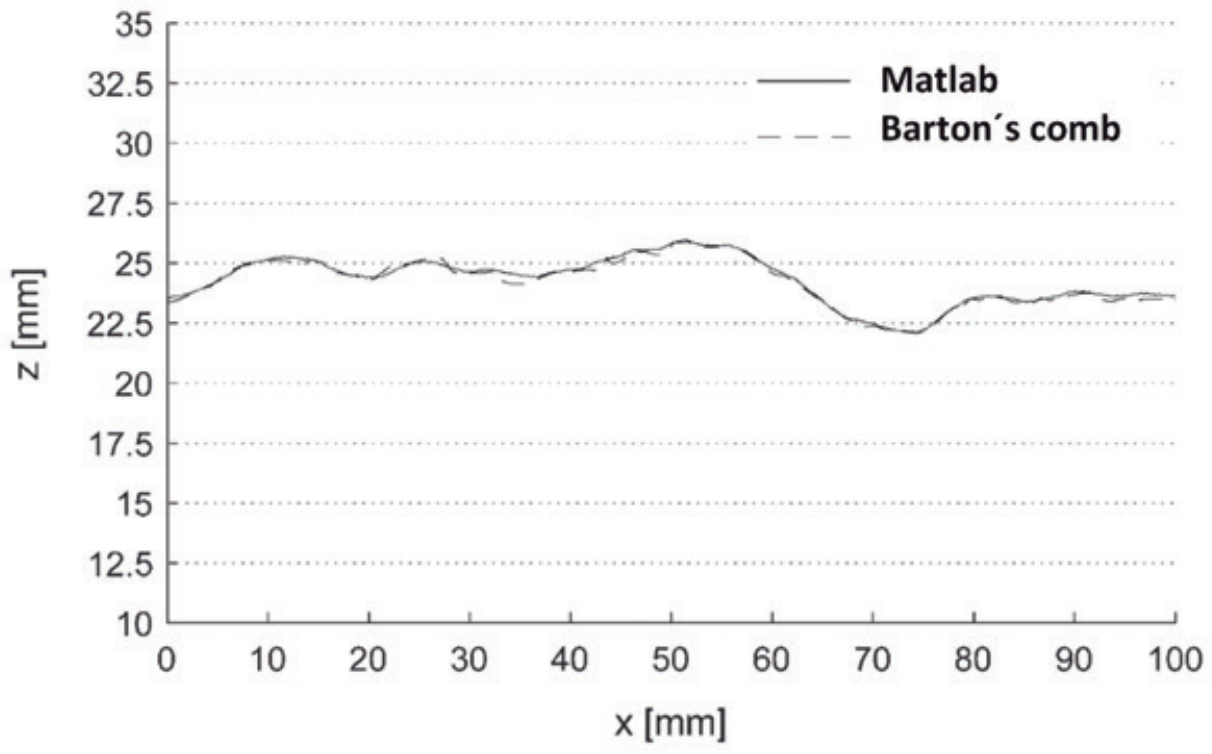

b)

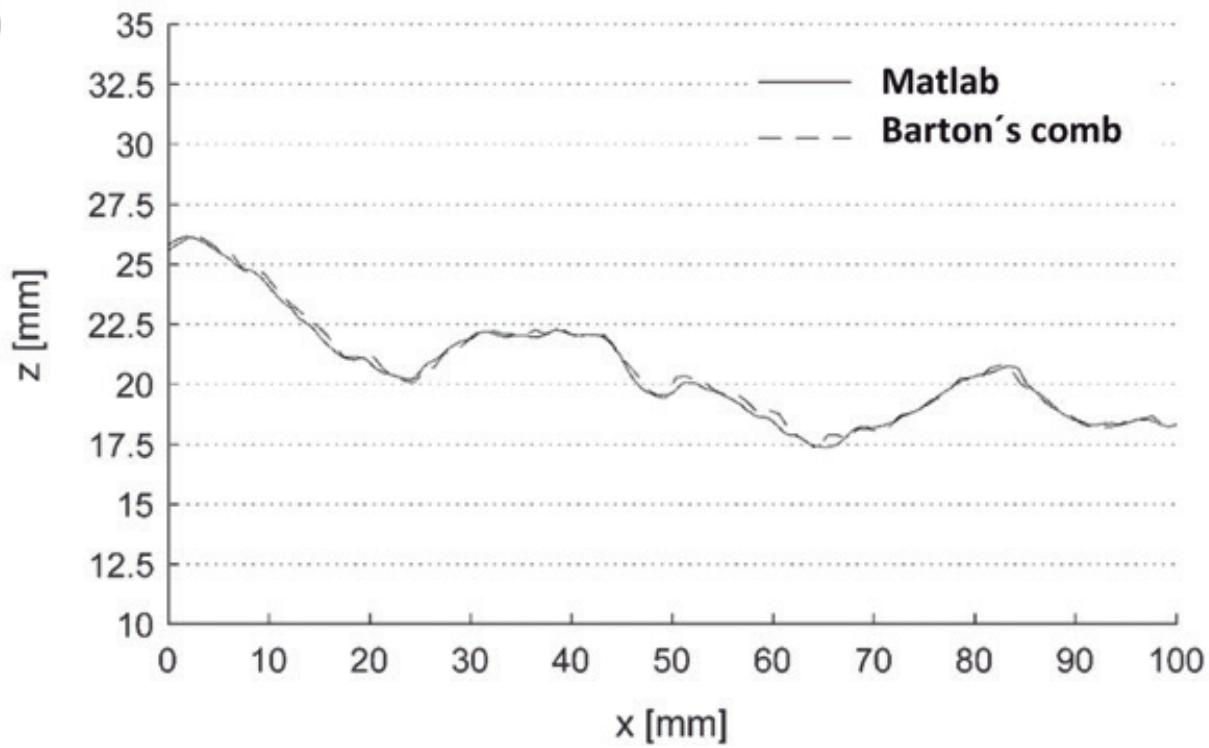

Figure 6. Examples of the comparison of profiles (extracted from the 3D point cloud and measured with Barton's comb): (a) Profile 54 (Sample 2); (b) Profile 29 (Sample 1).

Figura 6. Comparación de los perfiles extraídos de la nube de puntos $3 D$ y los medidos con el peine de Barton: (a) Perfil 54 (Muestra 2); (b) Perfil 29 (Muestra 1).

"Barton-comb" profile and the profile extracted from the 3D point cloud) at each location as an indicator of their similarity. $R_{a}$ is defined as:

$$
R_{a}=\frac{1}{L} \cdot \sum_{i=1}^{N}\left|z_{\mathrm{i}}\right| \cdot \Delta l
$$

where $z$ and $N$ were defined previously, $L$ is the profile length and $\Delta l$ is the horizontal distance between two sampled points.
If both profiles were identical, then $R_{a}$ would be theoretically zero. In our case, we obtained values of $R_{a}$ $<0.5 \mathrm{~mm}$ in all cases, with an average value for the 32 validation profiles considered for both samples of $\bar{R}_{a}=0.26 \mathrm{~mm}$.

Similarly, if we analyze both samples independently, we observe that the results are practically identical: $\bar{R}_{a}$ is equal to $0.26 \mathrm{~mm}$ for the 16 validation profiles of both Model 1 and Model 2, and the correspond- 


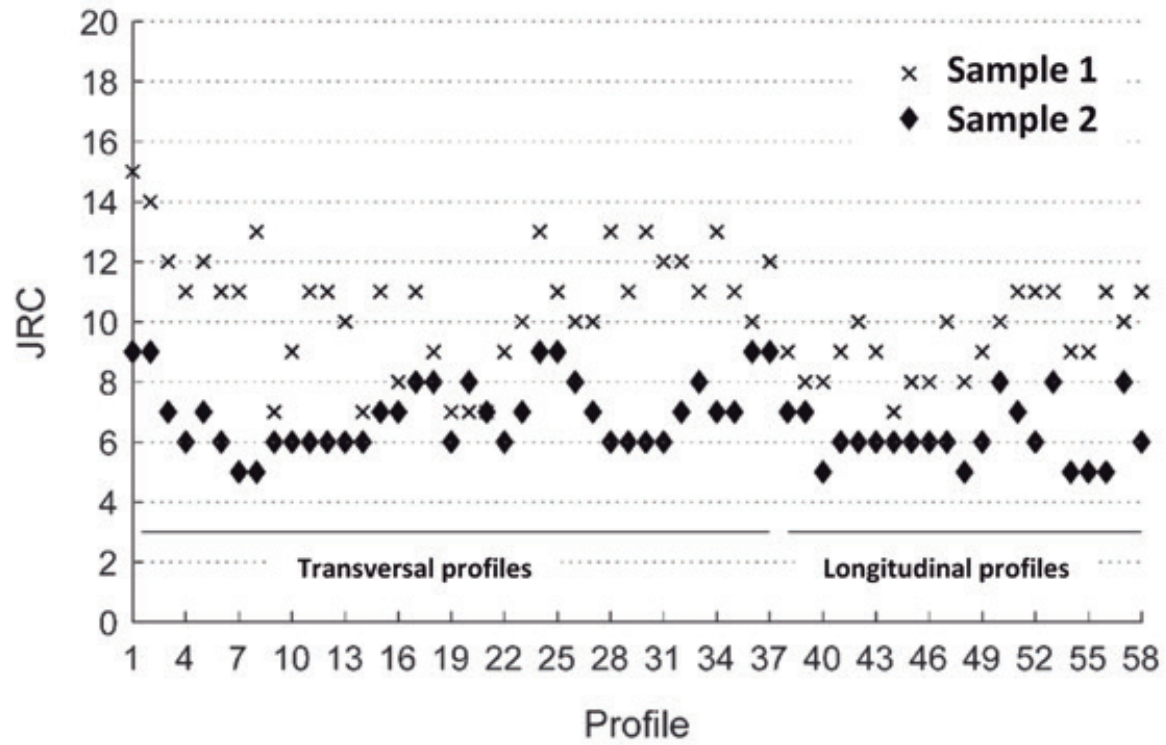

Figure 7. JRC values (estimated using correlations with $\mathrm{Z}_{2}$ ) for all the profiles considered.

Figura 7. Valores JRC (estimados mediante correlaciones con $Z_{2}$ ) para todos los perfiles empleados en el análisis.

ing standard deviations are also approximately equal $10.099 \mathrm{~mm}$ for Model 1, with the high quality dense point cloud; and $0.107 \mathrm{~mm}$ for Model 2, with the medium quality dense point cloud). This suggests that dense point clouds with the highest quality might not be necessary for this type of analysis, as they require much higher generation times whilst providing results of approximately the same quality.

\section{Roughness analysis}

\section{Estimation of JRC}

We have used the Joint Roughness Coefficient (JRC) to assess the adequacy of the proposed methodology for practical applications. The JRC is a simple (and subjective) tool commonly used to characterize the strength of rock discontinuities which summarizes the information about joint roughness into a unique parameter.

To that end, first we assigned a JRC value to all the profiles considered (i.e., to both the basic and validation profiles). To make the procedure more objective, we have used one relationship between JRC and one of the statistical parameters proposed to estimate joint roughness (see e.g., Tatone, 2009). For instance, $\mathrm{Li}$ and Zhang (2015) analyzed 47 equations from the literature correlating JRC and such parameters, con- cluding that correlations with $Z_{2}$ the statistical parameter (i.e., the average quadratic slope of the profile) or with $\sigma_{i}$ (the standard deviation of the average angle) tend to perform better. Here, we have used the correlation between the $Z_{2}$ statistical parameter (Equation 4 ) and JRC proposed by these authors.

$$
Z_{2}=\left[\frac{1}{L} \cdot \sum_{i=1}^{N-1} \frac{\left(Z_{\mathrm{i}+1}-Z_{\mathrm{i}}\right)}{\left(X_{\mathrm{i}+1}-X_{\mathrm{i}}\right)}\right]^{1 / 2}
$$

where $x_{i}$ and $z_{i}$ represent the coordinates of the $N$ equally-distanced sampling points selected at the joint surfaces, and $L$ is the profile length.

The correlations between JRC and $Z_{2}$ proposed by Li and Zhang (2015) are:

$$
\begin{aligned}
& J R C=55.7376 \cdot Z_{2}-4.1166 \\
& J R C=98.718 \cdot Z_{2}^{1.6833}
\end{aligned}
$$

Equation [5] is a linear expression proposed for $Z_{2} \in[0.074-0.433]$ and Equation [6] is an exponential expression that was found to perform better for planar or sub-planar joints with $Z_{2} \in[0-0.387]$. Since $Z_{2}$ depends on the sampling frequency (i.e., as the distance between points considered on the profile), Li and Zhang 


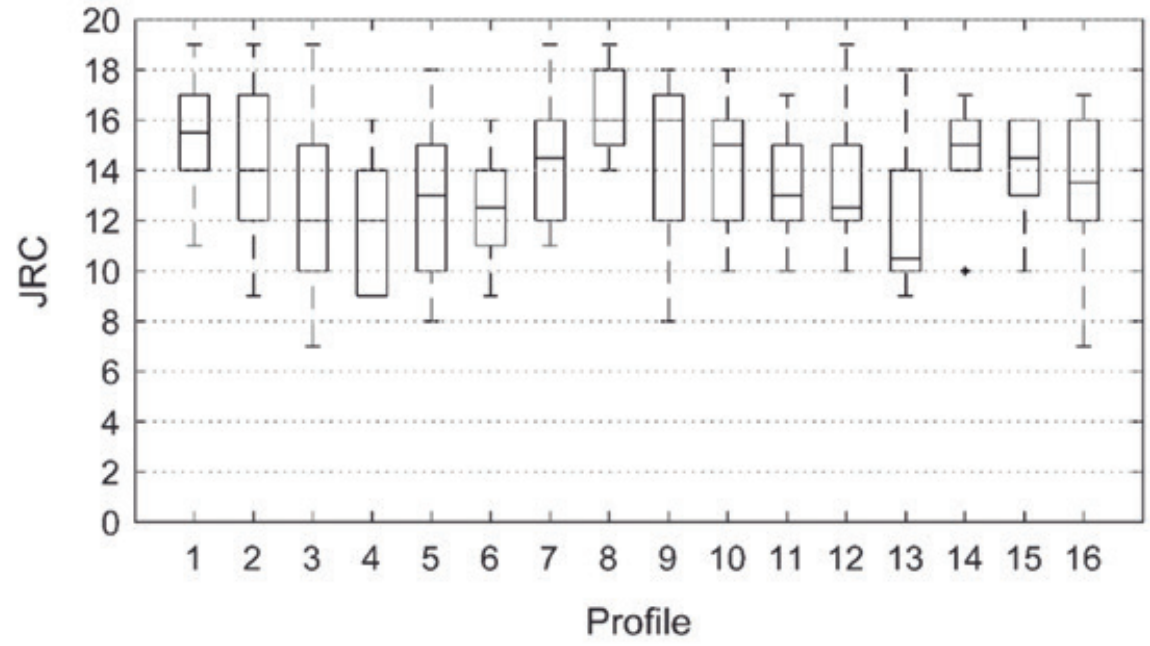

Figure 8. Summary of JRC values (estimated visually by 14 different people) for the 16 validation profiles considered for Sample 1.

Figura 8. Resumen de los valores JRC (estimados visualmente por 14 personas diferentes) para los 16 perfiles de validación considerados para la Muestra 1.

(2015) indicate that these expressions are valid for a sampling distance of $0.4 \mathrm{~mm}$. For this reason, to calculate the JRC using $Z_{2}$, we have extracted profiles from the $3 \mathrm{D}$ point cloud using a sampling frequency of $2.5 \mathrm{~mm}^{-1}$.

We have used the method described above to estimate the JRC values of all the 58 profiles considered in each sample. Figure 7 shows our results; Sample 1 produces JRC values between 7 and 15 in the transversal direction and between 7 and 11 in the longitudinal direction, whereas Sample 2 produces JRC values of 5-9 (transversal) and 5-8 (longitudinal). (Note also that the profiles shown in Figure 6 produced JRC values of 5 and 11.)

We have also used the 16 validation profiles of Sample 1 characterized with Barton's comb to compare their visually estimated JRC values with those obtained with the proposed approach using SfM and correlations with $Z_{2}$. To that end, a survey was conducted amongst 14 people, all with a geological or geotechnical education, and with different levels of experience and background, who were asked to assign JRC values to each profile, using the traditional approach of visual comparison against a set of reference profiles (Barton and Choubey, 1977). Figure 8 shows the results of this survey, clearly showing that visually estimated JRC values tend to be significantly higher than those estimated using the proposed approach, and that the visual approach is more subjective than the proposed one, with differences between observers of up to 12 points.

\section{Representing joint profiles using their spectral frequencies}

Following the recommendations by Shirono and Kulatilake (1997), we sampled the profiles obtained from the $3 \mathrm{D}$ model using a frequency of $10 \mathrm{~mm}^{-1}$ to carry out the spectral analysis. This allows us to obtain the spectral frequencies of each profile up to $5 \mathrm{~mm}^{-1}$, or one half of the sampling frequency. Figure 9 shows two examples of the frequency spectra calculated by means of the Fast Fourier Transform, for the two profiles presented in Figure 6 . The reader can note that the first profile (Figure 6(a) and 9(a)) is less rough, so that its spectrum decays rapidly to very low amplitudes for frequencies higher than $0.2 \mathrm{~mm}^{-1}$ and that its practically zero above $0.4 \mathrm{~mm}^{-1}$. Similarly, the other profile is rougher, and still has some frequency content (i.e., amplitudes higher than zero) for frequencies above $2 \mathrm{~mm}^{-1}$.

The next step, that needs to be carried out before the parametrization of the spectrum, is to establish the threshold between primary and secondary roughness or, in other words, between waviness and smoothness. Following Palmström (2001), we have established a frequency threshold of $0.5 \mathrm{~mm}^{-1}$, equivalent to a $2 \mathrm{~mm}$ distance that represents $1 / 50$ of the profile length. Once such a threshold has been established, it is straightforward (using a minimum squares approach) to obtain the parameters, $K, \lambda ; K_{1}, \lambda_{1}, K_{\mathrm{b}}, \lambda_{\mathrm{h}}$; or $p_{1}$ and $p_{2}$, of the best fit functions corresponding to the type of fitting approach proposed above (E, EEi, EEc, ESi; or ESc). 
a)

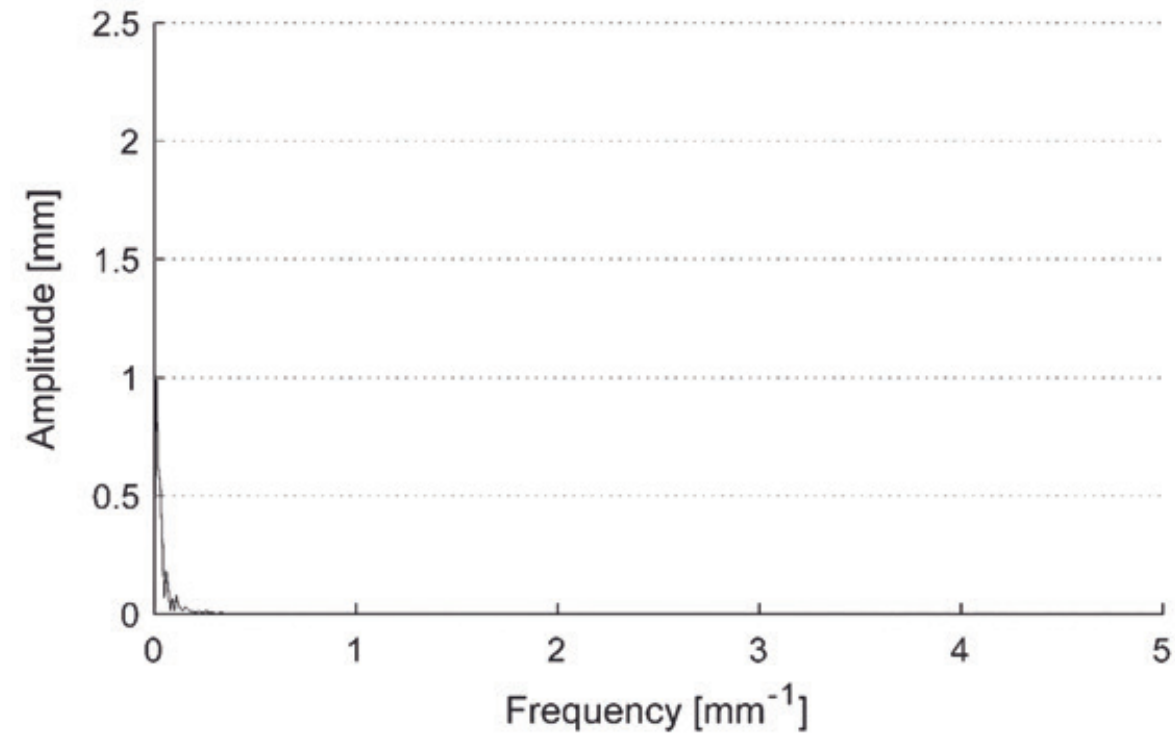

b)

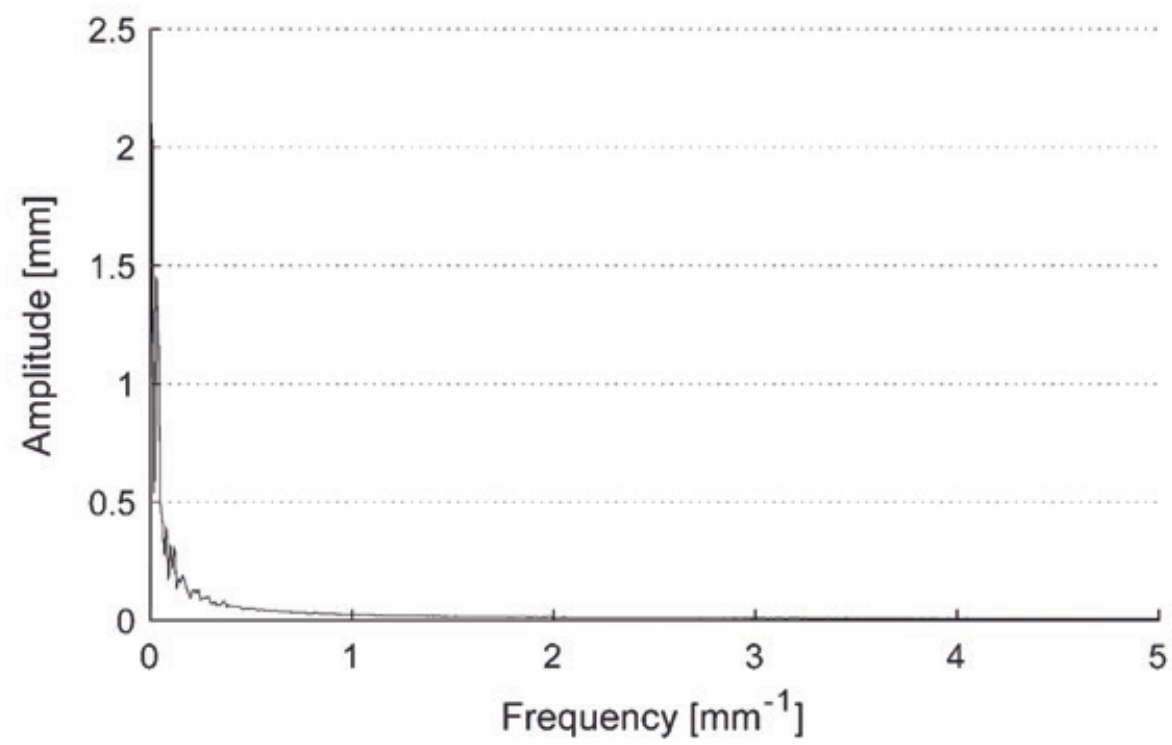

Figure 9. Examples of results of spectral analysis: (a) Profile 54 (Sample 2); (b) Profile 29 (Sample 1). (They correspond to the roughness profiles presented in Figure 6.).

Figura 9. Ejemplos de los espectros de frecuencia obtenidos: (a) Perfil 54 (Muestra 2); (b) Perfil 29 (Muestra 1). (Corresponden a los perfiles de rugosidad mostrados en la Figura 6.).

\section{Classification analysis}

To assess the performance of the different approaches considered to represent the frequency spectra of the roughness profiles, we have analyzed whether they could be successfully used to assess the roughness of each profile, as measured by its JRC. We used the JRC values estimated using their correlations with the $Z_{2}$ statistic, as results suggest that this approach is more robust than visual observation. In other words, we analyzed whether the JRC values of each profile could be estimated using the parameters obtained from the spectral analysis or not.

As just defined, this is a classification problem, in which the fitting parameters are the attributes, and where the JRC values represent the categories or 
JRC (Predicted)

\begin{tabular}{|c|c|c|c|c|c|c|c|c|c|c|c|c|c|c|c|c|c|c|c|c|}
\hline & 1 & 2 & 3 & 4 & 5 & 6 & 7 & 8 & 9 & 10 & 11 & 12 & 13 & 14 & 15 & 16 & 17 & 18 & 19 & 20 \\
\hline 1 & 0 & 0 & 0 & 0 & 0 & 0 & 0 & 0 & 0 & 0 & 0 & 0 & 0 & 0 & 0 & 0 & 0 & 0 & 0 & 0 \\
\hline 2 & 0 & 0 & 0 & 0 & 0 & 0 & 0 & 0 & 0 & 0 & 0 & 0 & 0 & 0 & 0 & 0 & 0 & 0 & 0 & 0 \\
\hline 3 & 0 & 0 & 0 & 0 & 0 & 0 & 0 & 0 & 0 & 0 & 0 & 0 & 0 & 0 & 0 & 0 & 0 & 0 & 0 & 0 \\
\hline 4 & 0 & 0 & 0 & 0 & 0 & 0 & 0 & 0 & 0 & 0 & 0 & 0 & 0 & 0 & 0 & 0 & 0 & 0 & 0 & 0 \\
\hline 5 & 0 & 0 & 0 & 0 & 0 & 1 & 0 & 0 & 0 & 0 & 0 & 0 & 0 & 0 & 0 & 0 & 0 & 0 & 0 & 0 \\
\hline 6 & 0 & 0 & 0 & 0 & 0 & 2 & 2 & 1 & 0 & 0 & 0 & 0 & 1 & 0 & 0 & 0 & 0 & 0 & 0 & 0 \\
\hline 7 & 0 & 0 & 0 & 0 & 1 & 1 & 0 & 2 & 1 & 0 & 0 & 0 & 0 & 0 & 0 & 0 & 0 & 0 & 0 & 0 \\
\hline 8 & 0 & 0 & 0 & 0 & 0 & 0 & 1 & 1 & 1 & 1 & 0 & 0 & 0 & 0 & 0 & 0 & 0 & 0 & 0 & 0 \\
\hline 9 & 0 & 0 & 0 & 0 & 1 & 1 & 0 & 1 & 2 & 0 & 0 & 0 & 0 & 0 & 0 & 0 & 0 & 0 & 0 & 0 \\
\hline 10 & 0 & 0 & 0 & 0 & 1 & 0 & 0 & 1 & 0 & 0 & 1 & 0 & 0 & 0 & 0 & 0 & 0 & 0 & 0 & 0 \\
\hline 11 & 0 & 0 & 0 & 0 & 1 & 0 & 0 & 0 & 0 & 1 & 2 & 0 & 1 & 0 & 0 & 0 & 0 & 0 & 0 & 0 \\
\hline 12 & 0 & 0 & 0 & 0 & 0 & 0 & 0 & 0 & 0 & 0 & 1 & 1 & 0 & 0 & 0 & 0 & 0 & 0 & 0 & 0 \\
\hline 13 & 0 & 0 & 0 & 0 & 0 & 0 & 0 & 0 & 0 & 0 & 0 & 0 & 0 & 0 & 0 & 0 & 0 & 0 & 0 & 0 \\
\hline 14 & 0 & 0 & 0 & 0 & 0 & 0 & 0 & 0 & 0 & 0 & 0 & 0 & 0 & 0 & 0 & 0 & 0 & 0 & 0 & 0 \\
\hline 15 & 0 & 0 & 0 & 0 & 0 & 0 & 0 & 0 & 0 & 1 & 0 & 0 & 0 & 0 & 0 & 0 & 0 & 0 & 0 & 0 \\
\hline 16 & 0 & 0 & 0 & 0 & 0 & 0 & 0 & 0 & 0 & 0 & 0 & 0 & 0 & 0 & 0 & 0 & 0 & 0 & 0 & 0 \\
\hline 17 & 0 & 0 & 0 & 0 & 0 & 0 & 0 & 0 & 0 & 0 & 0 & 0 & 0 & 0 & 0 & 0 & 0 & 0 & 0 & 0 \\
\hline 18 & 0 & 0 & 0 & 0 & 0 & 0 & 0 & 0 & 0 & 0 & 0 & 0 & 0 & 0 & 0 & 0 & 0 & 0 & 0 & 0 \\
\hline 19 & 0 & 0 & 0 & 0 & 0 & 0 & 0 & 0 & 0 & 0 & 0 & 0 & 0 & 0 & 0 & 0 & 0 & 0 & 0 & 0 \\
\hline 20 & 0 & 0 & 0 & 0 & 0 & 0 & 0 & 0 & 0 & 0 & 0 & 0 & 0 & 0 & 0 & 0 & 0 & 0 & 0 & 0 \\
\hline
\end{tabular}

Figure 10. Confusion matrix for the EEc approach.

Figura 10. Matriz de confusión para el ajuste EEC.

\begin{tabular}{cccc}
\hline \multirow{2}{*}{ Fitting approach } & \multicolumn{3}{c}{ Successful estimations (\%) } \\
\cline { 2 - 4 } & JRC & JRC \pm 1 & JRC \pm 2 \\
\hline E & 12.5 & 53.1 & 81.3 \\
EEi & 21.9 & 56.3 & 71.9 \\
EEC & 25.0 & 62.5 & 81.3 \\
ESi & 15.6 & 46.9 & 78.1 \\
ESC & 12.5 & 53.1 & 81.3 \\
\hline
\end{tabular}

Table 5. Results of the validation exercises for classifications conducted with the different approaches considered herein.

Tabla 5. Resultados de los análisis de clasificación para los diferentes ajustes propuestos.

classes to be classified. To conduct the analysis, we used the nearest-neighbour algorithm (1-NN algorithm) as implemented in software WEKA (Hall et al., 2009). The 1-NN algorithm is based on the assumption that elements belonging to the same class will be close to each other, so that the classification of a new object is conducted depending on the class of its nearest neighbour.

The classifier is initially developed using the 42 basic profiles of each sample, and the remaining
16 profiles of each sample are used for validation. (Note that developing a classifier that uses information from both samples has allowed us to develop a classifier with a wider applicability, since both profiles have different ranges of roughness as defined by their JRC values.)

Figure 10 shows, as an example, the confusion matrix of one of the analysescarried out, the one corresponding to the EEc approach. It can be observed that, although the percentage of correct estimations is relatively low (only about $25 \%$ ), the matrix is clearly diagonal, so that the percentage of successful estimations increases to $63 \%$ if we accept as valid one class immediately above or below the right answer (i.e., for a profile with JRC $=5$ we accept as valid if the profile was classified as $\mathrm{JRC}=4,5$ or 6 ); and to $81 \%$ if we accept as valid two classes above or below. Table 5 summarizes the results of this analysis for all the parametrizations considered $(E, E E i, E E c, E S i$, or ESc). Our results suggest that the parametrization provided by Approach EEc, i.e., considering two negative exponential functions (one for low frequencies, and other for high frequencies) and imposing their 
continuity at the threshold between low and high frequencies, has the best predictive performance in this case. Our results, however, are similar for several approaches, and further research is needed to identify the best approach for a wide generality of cases (i.e., different rock or joint types, with different roughness profiles due to their origin, etc.).

\section{Conclusions}

We propose a novel methodology to characterize the roughness of rock joints using an adequate parametrization of their frequency spectra. The approach commences with the development of (medium quality) 3D dense point clouds using the Structure from Motion (SfM) methodology. The main advantage of SfM over LiDAR is economical, as it has removed the need for special devices, and only a conventional camera and a SfM software are needed. Specific profiles can be extracted from the 3D model so that they can be analyzed after they are exported to a mathematical software such as MATLAB. Such profiles can also be analyzed using a frequency approach, and their frequency spectra can be obtained using, for instance, the Fast FourierTransform. Setting up a threshold between low and high frequencies (i.e., between waviness and smoothness, which control the shear behaviour of joints) has allowed us to test several approaches to represent the amplitude-frequency spectral relationships, and to obtain the best-fitting parameters for each case.

We have also developed a classifier to estimate the Joint Roughness Coefficient (JRC) of joints using the best-fitting parameters corresponding to each case. (The classifier is validated by comparison with JRC values estimated using existing correlations with the $Z_{2}$ statistical parameter.) Results suggest that JRC values estimated using the spectral information tend to be quite similar (with errors less or equal to \pm 2 in about $80 \%$ of cases) to those estimated using $Z_{2^{\prime}}$ hence improving the visually estimated ones. The capability of the methodology to differentiate amongst different levels of roughness also opens up the possibility of characterizing the shear behaviour of joints by considering their waviness and smoothness independently. This will be the subject of our future research efforts.

\section{Acknowledgements}

This research has been funded by the Spanish Ministry of Economy and Competitiveness, under Grant Number BIA2015-69152-R. This support is gratefully acknowledged.

\section{References}

Barton, N. (1973). Review of a new shear-strength criterion for rock joints. Engineering Geology, 7 (4), 287-332.

Barton, N., and Choubey, V. (1977). The shear strength of rock joints in theory and practice. Rock mechanics, 10 (1), 1-54.

Chae, B.G., Choi, J.H., Ichikaea, Y., and Seo, Y.S. (2011). Analysis of the permeability characteristics along roughwalled fractures using a homogenization method. Nuclear Engineering and Technology, 44 (1), 43-52.

Ge, Y., Kulatilake, P. H., Tang, H., and Xiong, C. (2014). Investigation of natural rock joint roughness. Computers and Geotechnics, 55, 290-305.

Hall, M., Frank, E., Holmes, G., Pfahringer, B., Reutemann, P., and Witten, I.H. (2009). The WEKA Data Mining Software: An Update. SIGKDD Explorations, vol. 11, № 1.

ISRM Commission on Standardization of Laboratory and Field Tests (1978) 'Suggested Methods for the Quantitative Description of Discontinuities', International Journal of Rock Mechanics and Mining Sciences and Geomechanics, 15, 319-368.

Li, Y., and Huang, R. (2015). Relationship between joint roughness coefficient and fractal dimension of rock fracture surfaces. International Journal of Rock Mechanics and Mining Sciences, 75, 15-22.

Li, Y., and Zhang, Y. (2015). Quantitative estimation of joint roughness coefficient using statistical parameter. International Journal of Rock Mechanics and Mining Sciences, 77, 27-35.

LLC, A. (2016). Agisoft PhotoScan User Manual-Professional Edition. ed. St.Petersburg, Russia. Version 1.2.

Palmström, A. (2001). Measurement and characterization of rock mass jointing. Chapter 2 of the book: In-Situ Characterization of Rocks, A.A. Balkema Publishers.

Patton, F.D. (1966). Multiple modes of shear failure in rock. Proc. 1st Int. Congress on rock Mechanics, Lisbon, vol. 1, pp. 509-513.

Prandoni, P., and Vetterli, M. (2008). Signal processing for communications, EPFL Press.

Shirono, T., and Kulatilake, P.H.S.W. (1997). Accuracy of the spectral method in estimating fractal/spectral parameters for self-affine roughness profiles. International Journal of Rock Mechanics and Mining Sciences, 34 (5), 789-804.

Slama, C.C., Theurer, C., and Henriksen, S.W. (1980). Manual of Photogrammetry. American Society of photogrammetry. Falls Church, Va., United States.

Tatone, B. (2009). Quantitative characterization of natural rock discontinuity roughness in-situ and in the laboratory. MasterThesis, University of Toronto.

Tatone, B. S., and Grasselli, G. (2013). An investigation of discontinuity roughness scale dependency using high-resolution surface measurements. Rock mechanics and rock engineering, 46 (4), 657-681. 
The MathWorks, Inc. (2019). MATLAB (MATrix LABoratory), Version 9.6 (R2019a).

Tse, R., and Cruden, D.M. (1979). Estimating joint roughness coefficients. International Journal of Rock Mechanics, 16 (5), 303-307.

Unal, M., and Unver, B. (2004). Characterization of rock joint surface degradation under Shear Loads. International journal of Rock Mechanics and Mining Science, 41 (3), 145-150.

Wernecke, C., and Marsch, K. (2015). Mapping rock surface roughness with photogrammetry. In ISRM Region- al Symposium-EUROCK 2015. International Society for Rock Mechanics and Rock Engineering.

Yang, Z.Y., Di, C.C., and Yen, K.C. (2001). The effect of asperity order on the roughness of rock joints. International Journal of Rock Mechanics and Mining Science, 38 (5), 745775.

Zou, L., Jing, L., and Cvetkovic, V. (2015). Roughness decomposition and nonlinear fluid flow in a single rock fracture. International Journal of Rock Mechanics and Mining Sciences, 75, 102-118.

Recibido: julio 2019

Revisado: noviembre 2019

Aceptado: enero 2020

Publicado: marzo 2021 